\title{
Apport des technologies numériques à l'étude des fortifications du génie militaire français dans une ville d'Algérie au début de la colonisation : Djidjelli, 1839-1862 \\ ${ }^{a}$ Mustapha Blibli, a Ammar Bouchair, ${ }^{c}$ Faouzi Hannouf \\ ${ }^{a}$ Université de Jijel- Laboratoire de recherches CBE, Jijel, Algerie, musblibli@gmail.com, abouchair@gmail.com, \\ ${ }^{\mathrm{b}}$ Architecte libéral, Paris, France, faouzi.hannouf@gmail.com.
}

\begin{abstract}
Le point de départ de ce travail était de combler un manque cruel dans l'histoire de la ville de Djidjelli, au tout début de sa colonisation, du moins pour ce qui concerne sa transformation, période méconnue des jijeliens actuels eux-mêmes - et pourtant une des plus structurantes de son histoire.

Se basant sur les travaux de certains auteurs et sur les deux fonds d'archives qui conservent en France des documents relatifs à l'Algérie ; ce sont le Centre National des archives d'Outre-Mer (CAOM à Aixen-Provence, archives nationales) et le Service Historique de la Défense (SHD à Vincennes). Parmi tous les dossiers, ceux parmi lesquels on peut trouver les documents qui concernent Djidjelli.

Notre travail consistera en la sélection et la digitalisation des principales cartes des archives de la défense en vue de la modélisation $2 \mathrm{~d}$ et $3 \mathrm{~d}$ de l'intervention du génie militaires et des fortifications.

La méthode développée permet de résoudre et de générer des volumétries urbaines plausibles dans les cas les plus fréquents. Le modèle 3D obtenu, malgré sa simplicité géométrique, permet de visionner la cité et ses fortifications sous différents angles, aussi il sera possible de retrouver les traces de ces fortifications dans la ville actuelle ce qui permettra de comprendre la configuration actuelle de la ville et d'ouvrir aussi de nouvelles pistes de recherches en histoire, architecture et urbanisme.
\end{abstract}

Keywords : Génie militaire, fortifications, modélisation 2d et 3d, histoire urbaine.

\section{Introduction}

Notre travail en premier lieu consiste à retracer l'intervention du génie militaire français sur le territoire de la ville de Djidjelli à travers toutes les étapes depuis 1839 jusqu'à 1856, une manière de comprendre cette intervention et ses phases, les archives de la défense et ceux d'outre-mer représentent la base et le support de cette recherche qui va nous aider à traduire ces données par des outils numériques sous forme d'une maquette numérique capable de synthétiser le toutes ces données, après la présentation de la ville de djidjelli, son histoire et un bref aperçus sur les interventions du génie militaires représentées par les archives historiques de la défense, nous exposerons les outils et programmes utilisés dans modélisation $2 \mathrm{~d}$ et $3 \mathrm{~d}$ ainsi que la méthode pour la réalisation d'une maquette numériques qui deviendra à son tour un outil de ; présentation ; vérification, collaboration ; vulgarisation et formations dans le domaine de l'histoire, l'architecture et l'urbanisme.

\subsection{Aperçu historique}

La ville de Djidjelli depuis sa création, a subi des dominations successives de toutes les 
civilisations qu'a connu l'histoire de la méditerranée. Toutes ces civilisations avaient pour intérêt sa position stratégique, et sa morphologie formant des havres naturels. La vie s'est développée autour de son littoral, c'est à partir de ce lieu que s'est établie la croissance de la ville.

La ville traditionnelle occupait principalement la presqu'île séparée de la mer par un petit espace étroit, qu'une vieille muraille protégeait depuis des siècles contre les attaques des Kabyles

Avec les phéniciens, l'occupation s'est limitée à un espace très réduit, les monuments attestant leur présence sont des tombeaux creusés dans la roche, qui sont encore visible à la «Pointe noire » (actuellement Rabta).

Pour les romains, la ville n'était pas restreinte à la presqu'île de la citadelle actuelle, elle s'étendait sur l'emplacement de la ville moderne actuelle, surtout dans la partie qui borde la mer (le port).

L'occupation Byzantine s'est caractérisée par l'élargissement de l'ancienne ville et sa fortification d'une muraille jalonnée de tourelles, par contre la succession de l'invasion vandale, n'a laissé aucune ruine de cette époque.

Pendant l'époque arabe aucun grand changement n'a été opéré, les remparts romano byzantins ont été conservés a l'instar des villes médiévales algériennes (Bouchair, Dupagne, 2003), il y a eu seulement la création des édifices de cultes, qui seront détruits ultérieurement par les normands, au même titre que le rétrécissement de l'enceinte de la ville.

A l'arrivée des turcs, ils occupèrent l'ancienne enceinte. Cette période est marquée par la mise en place des structures d'échange et de rencontre, places, marché, mosquée, port...etc.

Le port se situait dans la baie qui s'ouvre à l'Est, protégé ainsi des vents d'Ouest par la terre, du nord par une ligne de récifs (barrière insuffisante contre les grosses mers), et une jetée couvrant le coté Est de la rade.

Suite à l'expédition du Duc de Beaufort en 1664, La ville a été totalement détruite par les bombardements. Avec l'arrivé des français, et suite à une série d'affrontement, L'attention des envahisseurs se porta alors sur la ville qui était dans un état de vétusté avancée.

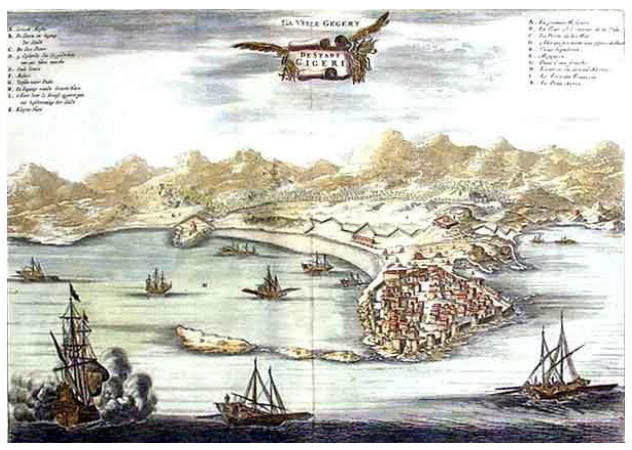

Fig. 1- Carte de la ville de DJIDJELLI et ses environs (Jean Ogylby, 1670)

On mit à profit de vieilles fortifications en ruines. On restaura l'ancienne tour hexagonale qui prit le nom du fort de St Ferdinand, au centre fut établi une redoute carrée, le Fort Galbois relié au fort St Eugénie. On décida de relever sous le nom du Fort Duquesne des ruines d'un ancien établissement musulman.

\section{Interventions du génie militaire}

\subsection{Prise de possession : sécuriser, s'installer, s'équiper et réorganiser la ville (1839-1854 env.)}

Apres la prise de possession de djidjelli, la première des choses a faire était de sécuriser le territoire autour de la cité par l'établissement d'une série de postes avancés, ce dispositif comprenait aussi un système de communication reliant les forts entre eux et avec la ville.

Ce dispositif a nécessite des réorganisations afin de remédier a certaines défaillances.

D'un autre côté, a sa prise la vielle ville de djidjelli implanté sur la presque ile rocheuse était dans un état délabré, son enceinte d'origine byzantine était en ruines, ou des travaux de crépissage et d'agrandissement des fortifications ont été entrepris. La ville aussi a fait l'objet d'interventions comme l'assainissement et la restructuration de la ville ou on a percé, aligné, nivelé et pavé les rues. On a établi la 
classification de la voirie ainsi que la mise à jour $\mathrm{du}$ plan de distribution, d'alignement et de nivellement de la ville de djidjelli.

Pendant cette phase on a pensé aussi a aménager le territoire. Sa délimitation, l'assainissement et le dessèchement de la plaine. Des travaux de reboisement ont eu lieu ou on a planté plus de 1500 arbres.

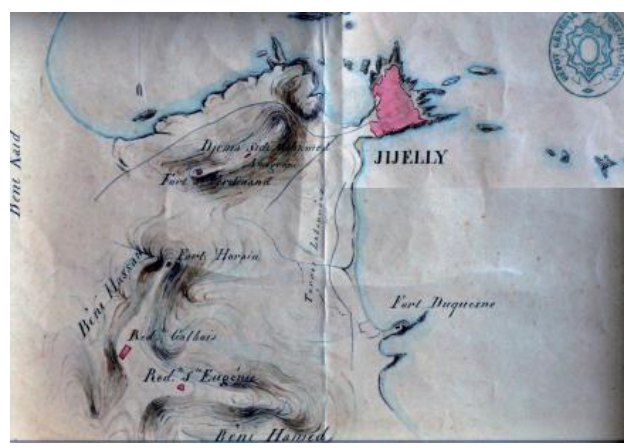

Fig.2- SHD, art.8, section1, Djidjelli, carton 1.

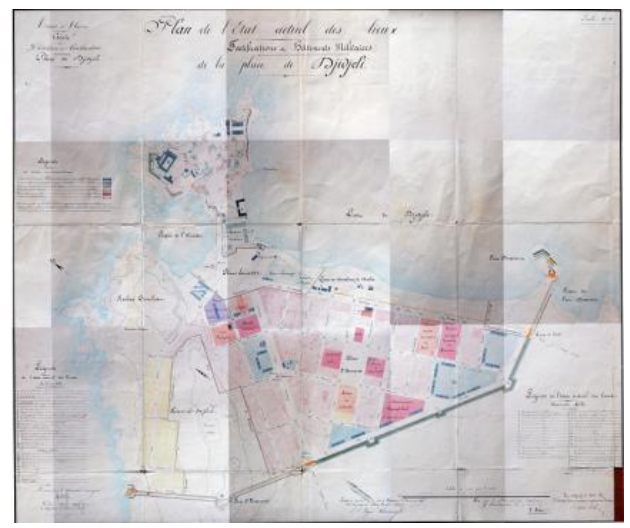

Fig. 3- Plan de l'état actuel des lieux de la place de Djidjelli.1957.

\subsection{Agrandissement de la ville: concevoir une nouvelle ville (1851-1856)}

La question d'agrandir la ville existait avant le tremblement de terre 1856, la population grandissante étouffait dans l'enceinte étroite de djidjelli et suite à l'expédition de Kabylie et la pacification des territoires environnant des relations commerciales entre les français et les tribus de la région commencèrent. Le commandant supérieur du Génie ainsi que le commandant du cercle, le commandant de la division, le chef du Génie et le directeur des Fortifications, pensent qu'il est maintenant temps de donner à la ville les moyens de son extension. Le Ministre de la Guerre autorise l'établissement d'un projet d'agrandissement pour la ville. Ce sont ici les intérêts de la colonisation qui sont en jeu. On entama l'étude $\mathrm{du}$ plan de distribution, d'alignement et de nivellement de la nouvelle ville de Djidjelli, et ses fortifications, le tremblement de terre du 2122 Aout 1856 ou l'ancienne vielle a été quasiment décimé ce qui accéléra le Transfer de la population civile sur le site de la nouvelle ville.

\subsection{Reconstruire la vieille ville en citadelle militaire (1857-1862)}

Les ingénieurs du Génie militaire s'occupaient d'agrandir la ville depuis 1854 . La nouvelle ville est en projet: à terme, elle deviendra le véritable centre de la population civile. La vieille ville lui servira sans doute, alors, de citadelle : elle forme le réduit de la position puisque quasiinaccessible sur ses abords. D'autre part, elle renferme la plus grande partie des établissements militaires.

Statut confirmé: quartier exclusivement militaire. Acquérir l'intégralité des terrains de l'ancienne ville:projets et contre-projets pour l'installation des établissements militaires Fortifier le nouveau quartier militaire. Entreprendre la fortification du nouveau quartier militaire:

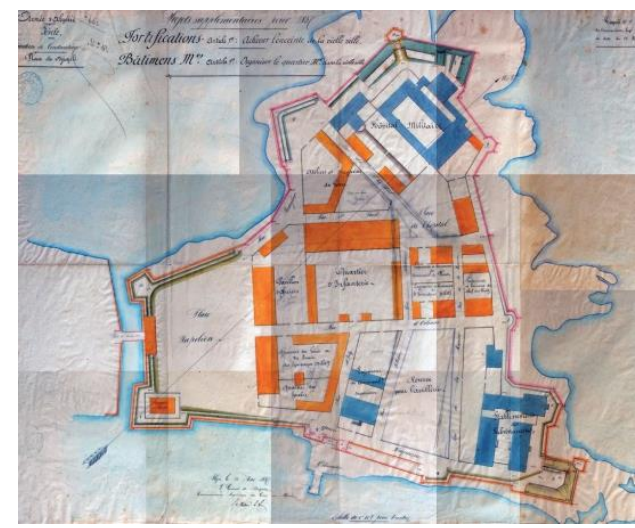

Fig. 4- Achever l'enceinte de la vieille ville \& Organiser le quartier militaire, 1957. 


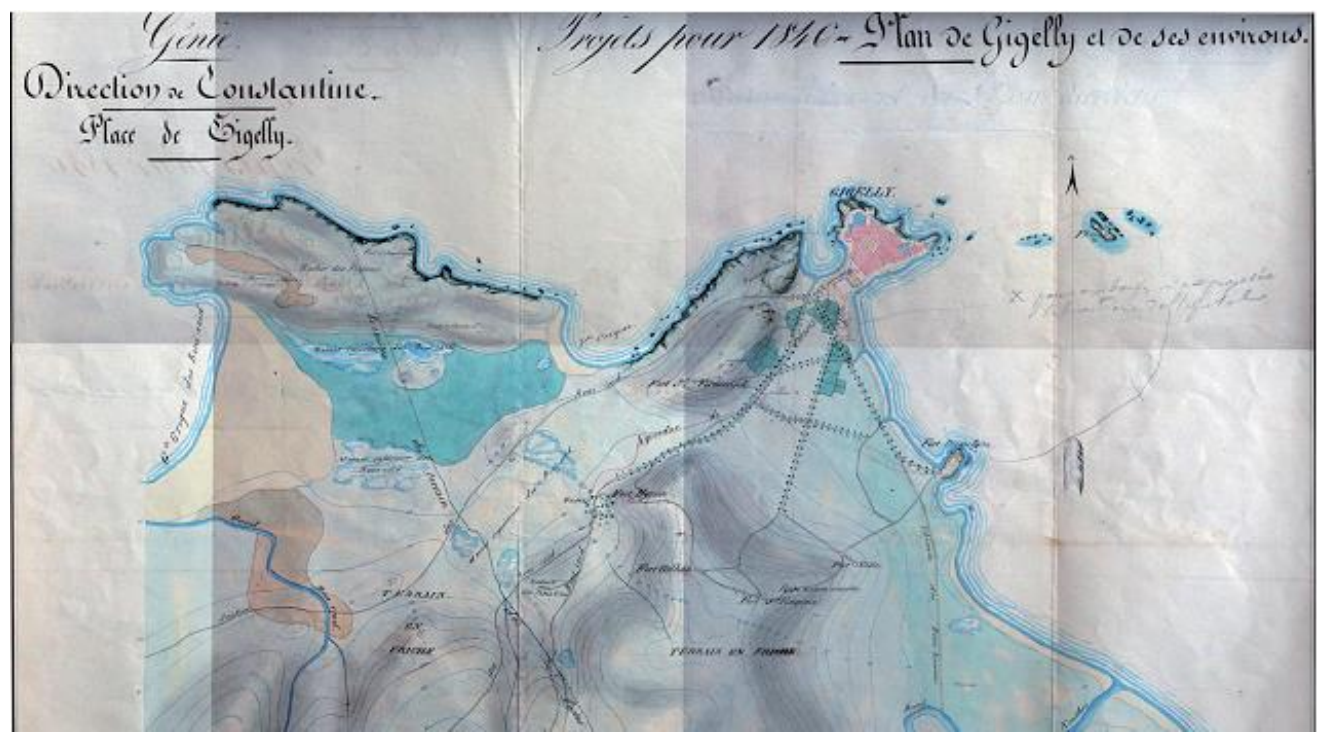

Fig. 5- Feuille de dessin unique des projets pour 1848 et 1849 : Plantation de 1500 arbres dans la plaine, CAOM, FM, F80, 810.

\section{Outils numériques et logiciels de CAO}

Dans cette phase de travail et après la sélection de toutes les cartes pertinentes, nous avons entamé la numérisation et la digitalisation de tous les plans du génie a notre disposition ainsi que tout autres supports, et ceci pour une meilleure exploitation, calage et superpositions, nous notons aussi le recours a des programmes de traitement et correction des images.

Mais en ce qui concerne la modélisation géométrique nous avons utilisé les logiciels de CAO disponibles sur le marché et dont nous avons l'habitude de travailler avec.

\subsection{La plateforme d'AutoCAD}

La plupart des agences d'architecture travaillent depuis longtemps avec le système informatique AutoCAD® Autodesk. Il est désormais un standard de l'industrie. Il a été continuellement adapté à la profession en améliorant ses outils ou en proposant d'autres fonctionnalités pour avoir une meilleure convivialité avec ses utilisateurs.

Les premières versions du logiciel (DAO) sont proposées pour aider le dessin, purement formel. Il a été basé par des concepts géométriques comme : la ligne, le point, le rectangle, l'arc, le cercle et le polygone qui seront inscrits dans un système cartésien.

Aujourd'hui, ce système a évolué et a trouvé des spécialisations par profession qui diffèrent dans la création personnalisée des objets paramétriques.

\subsection{La plateforme de SketchUP}

Le logiciel SketchUP (Trimble) est considéré comme « un des outils plus adapté aux concepteurs et à leurs habitudes figuratives ». Ce système de modélisation des données 3D (sketch $=$ esquisses) permet facilement une adaptabilité fonctionnelle aux usagers. Le logiciel est basé sur le principe de modélisation de masse. Une fois que la surface est créé, il est possible de la pousser ou de la tirer (extrusion) pour ensuite, réaliser des opérations booléennes aux volumes (modeleur surfacique) par le biais de la fonction Intersection.

Comme on a déjà cité, un objet $3 \mathrm{D}$ est un volume. SketchUP utilise entièrement ce fondement dans sa manière d'opérer. Il permet de créer des modèles réutilisables dans d'autres modèles. A partir de la fonction «Créer un composant », il est possible de concéder à un objet 3D des caractères individuels qui puissent 
se transférer d'un projet \{ un autre. Les entités composant sont des modèles dont ses attributs sont reproductibles. Au moment qu'ils sont créés, il est aussi probable de combiner plusieurs d'eux dans un seul système. Les entités groupe est l'association des composants.

Le choix s'est porté sur ces deux programmes essentiellement pour leur interopérabilité opérationnelle et la facilite d'échange et de transfert possibles entre eux dans un même système de modèles $2 \mathrm{~d}$ et $3 \mathrm{~d}$, ou nous avons remarqué le manque d'incompatibilité lors de l'utilisation entre les dessins bi. Et tridimensionnelles et ceci a cause de l'utilisation de programmes différents ou la possibilité d'échange entre eux consiste en des opérations d'import et export des fichiers.

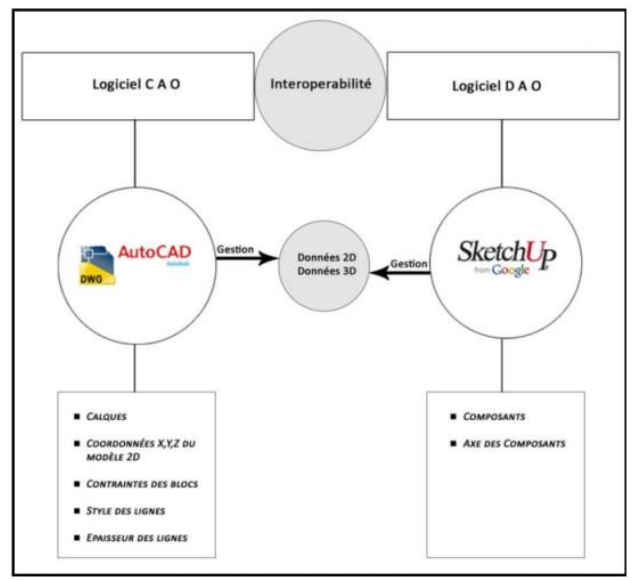

Fig. 6- Graphique de passage des données entre les logiciels CAO/DAO (AutoCAD/SketchUP)

\subsection{La modélisation bidimensionnelle}

La majeure partie de cette phase se fait sous plateforme AutoCad, on y intègre les cartes numérisées avec différentes méthodes, par la digitalisation, ou tout simplement par l'insertion des images raster et le retraçage des lignes directement. Avant toute chose il faut définir la géolocalisation des cartes et plans, les mettre à une échelle unique et cela on les superposant sur l'image satellite. Préparation du fichier suivant les calques qui seront nommés en fonctions des éléments définies soit le terrain, les voies, les constructions, les remparts....etc. ce qui facilitera cette phase de dessin en $2 d$, ou chaque carte correspond à un composant.

Par exemple le plan topographique sera redessiné sur un calque dénommé « cadre naturel », le plan de fortification et remparts sur un calques dénommé «fortifications » etc. à la fin nous aurons un fichier DWG ou DXF constitué de plusieurs calques correspondants aux éléments définissants notre site. Ce qui facilitera évidement l'étape suivante qui est la modélisation tridimensionnelle, aussi la conversion de chaque dessin de calque en un bloc dans le but qu'il soir reconnu dans SketchUp en tant que composant ce qui permet d'alléger et facilite les échanges tout en actualisant les changements opérés (mise à jour) entre les deux plateformes.

En ce qui concerne le calque du cadre naturel, nous recommandons de l'isoler dans un nouveau fichier, donner à chaque courbe de niveau son altitude mentionné sur le plan topographique, ceci rendra modélisation $3 \mathrm{~d}$ du cadre naturel et relief une opération très facile (voire section suivante).

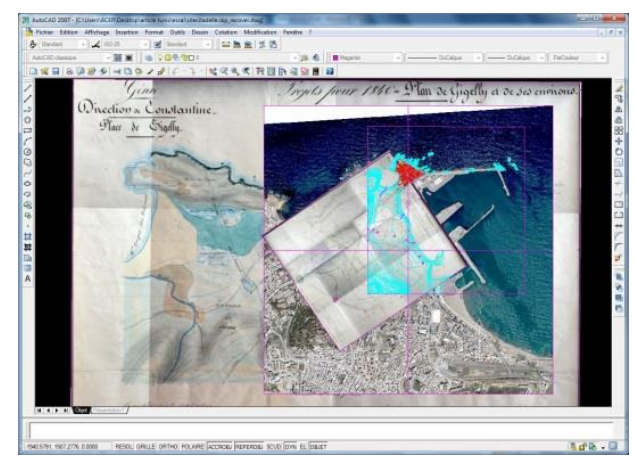

Fig. 7- Interface utilisateur du logiciel AutoCad: superposition et mise à l'échelle entre les cartes et l'image satellite actuelle.

\subsection{La modélisation tridimensionnelle}

Pour ce qui est de la modélisation tridimensionnelle $3 \mathrm{~d}$ dans le logiciel SketchUp, d'abord nous important le calque du cadre naturel qui est sous format dwg ou dxf, le travail qu'on a commencé déjà sous Autocad ' voire section précédente), avec l'outil «Bac à Sable» qui permet de générer 
automatiquement le relief et la topographie ( fig....).

En parallèle nous entamerons la modélisation des autres constituants de notre maquette qui représentent les différents calques importé et créés précédemment. Ce qui va nous aider à organiser et gérer cette phase, sur la base des élévations (profils, coupes, façades et détails techniques).

A la fin nous entamerons l'opération d'assemblage entre le cadre naturel et les composants bâtis, suivant la projection des tracés planimétrique sur le relief à l'aide de l'outil «Tampon» et l'insertion des volumes à leurs emplacements.
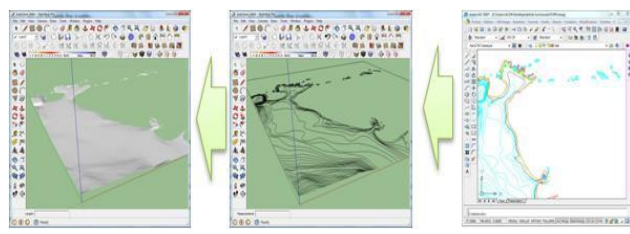

Fig. 8- transfert d'AutoCad vers SketchUp, modélisation du relief et topographie.

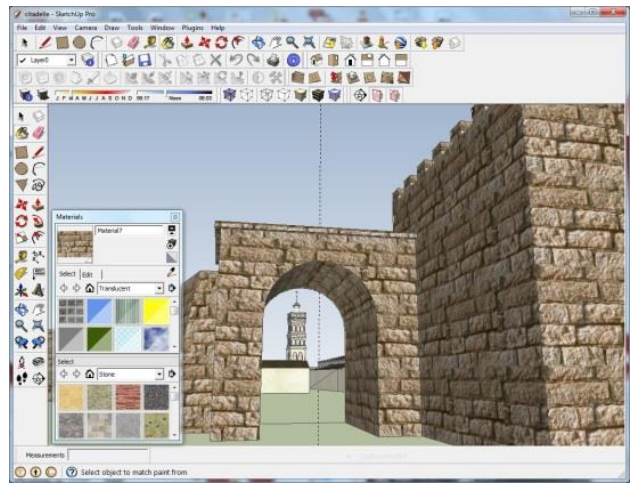

Fig.9- Interface utilisateur SketchUp, application des textures.

\subsection{Application des textures}

Dans cette phase intéressante du travail et un peu délicate en même temps, des recherches sur les matériaux de construction de l'époque et aussi sur le contexte historique, par le biais de collecte de dossiers et photographies des matériaux ou par la visite de vestiges et lieux analogues. Aussi les ambiances lumineuses des lieux en fonction de leur géolocalisation, d'un autre coté on peut ajouter des personnages, de la faune et la flore de l'époque susceptible de donner plus d'animation et de réalisme.

\subsection{Résultats de la modélisation}

Apres la finalisation du model 3d, nous aurons une maquette numérique qui permet de faire des ballades dans le territoire des anciennes fortifications, des comparaisons diachroniques, voire même des superposition avec l'état actuel de la ville, ceci dépend beaucoup du degrés de détail appliqué, le choix et le traitement des matériaux et textures, or avec l'introduction des détails des constructions, des espaces aussi l'ajout des modèles de personnages, objets et végétations, les rendus seraient plus satisfaisants et attractif, d'un autre coté on peut aussi établir des captures, des impression et des extrait vidéos pour d'éventuel usage.

- Projections orthogonales.

Elles consistent en des images et projection géométriques à exploiter dans les différentes études; les projections planimétriques, les élévations, façades, profils topographiques et détails.

- Vues et perspectives.

Elles représentent les vues générales et celles de détails sous n'importe quel angle de vue en fonction de l'objet à décrire du territoires des fortifications.

- Les extraits et films vidéo.

Dans la plus parts des programmes de modélisation tridimensionnelle, on trouve des outils d'enregistrement d'animation et ballades dans le modèle $3 \mathrm{~d}$, qu'on peut exporter sous forme de fichiers vidéos lisibles sur n'importe quels lecteurs multimédia.

\section{Conclusions}

Au bout de ce travail nous avons démontré la capacité des outils numériques à emmagasiner, synthétiser et diffuser l'information historique sous forme de présentation géométrique tridimensionnelle, cette dernière nous permet de visualiser des états et des situations historiques, 
les superposer à l'état actuel pour une comparaison diachronique ceci aide à saisir l'histoire urbaine de la ville de djidjelli. Dans le cas de cette étude, elle nous aide à comprendre les interventions du génie militaire dans son époque, comprendre la succession des étapes de l'occupation du territoire, mais aussi les traces urbains encore existant dans la ville actuelle, éclaircir certaines ambiguïtés sur certaines configurations spatiales.
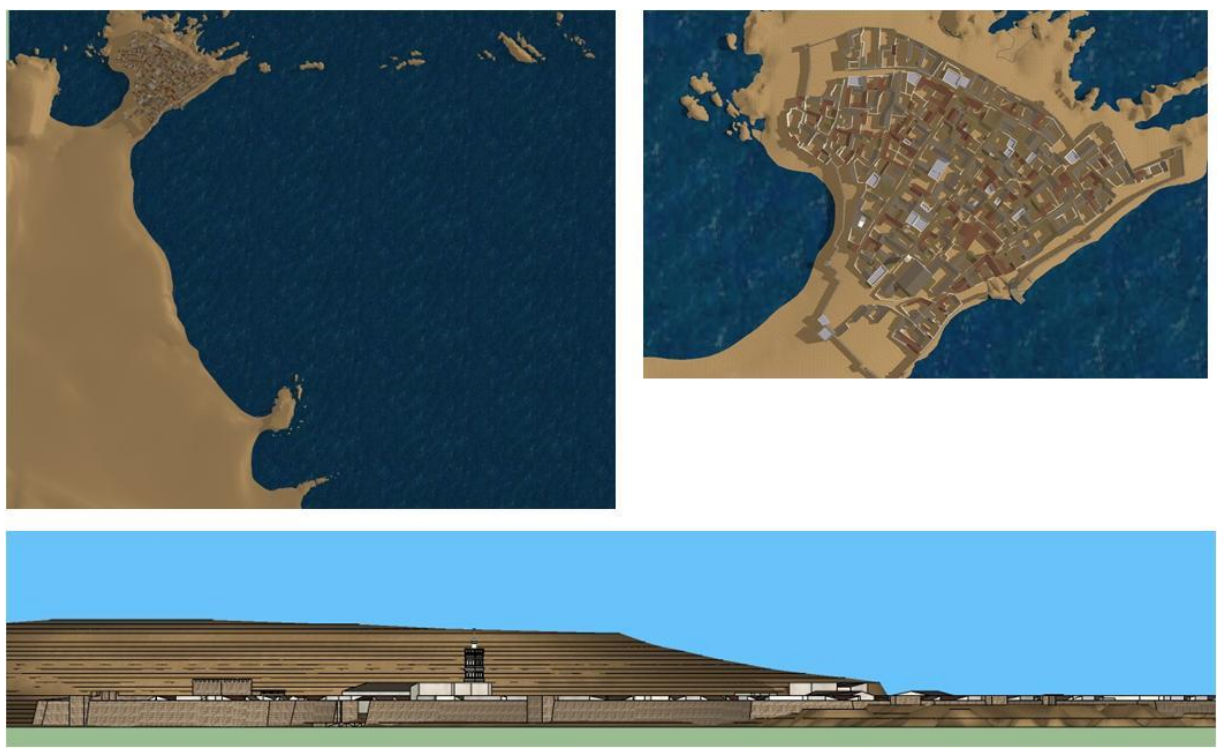

Fig. 10- Rendu et images; projection orthogonale et perspectives

\section{References}

AAVV. (s. d.) Places Françaises, 1680-1880 Environ : Mémoires Et Plans Des Projets Annuels De Travaux Effectués Aux Fortifications Et Bâtiments Militaire, Djidjelli, Carton 2: De 1845 À 1853.Vencennes, Paris.

Alkhoven, P. (2005). City Map To Virtual Reality : 3d Reconstruction Models Of Cities Gim Int., V. 19, N. 10.

Astruc, M. (1937). "Nouvelles Fouilles À Djidjelli, Algérie”, Revue Africaine, Alger, pp. 199-253.

Autodesk, (2010). Atelier Des Nouvelles Fonctionnalités. Autocad. France : S.N.

Bachelot, B. (2003) .Louis Xiv En Algérie : Gigeri, 1664, Monaco.

Blibli, M., Bouchair, A .Hannouf,F. (2012). "Three Dimensional Reconstitution of an Old Town from

Historical Documents: Case of the Medina of Jijel in Algeria", CAAD | INNOVATION | PRACTICE [6th International Conference Proceedings of the Arab Society for Computer Aided Architectural Design (ASCAAD 2012 / ISBN 978-99958-2-063-3], Manama (Kingdom of Bahrain), 21-23 February 2012, pp. 191; 285-303

Bouchair A., Dupagne A., (2003). Building Traditions of Mzab Facing theChallenges of Re-shaping of its Built form and Society, Building and Environment, 38(11), pp. 1345-1364. ISSN: 0360-1323. Elsevier. 
Coulais, J.F., Pinol, J.L. (2002). "La Reconstitution Historique Des Forms Urbaines" (A` Propos De Naples, San Francisco Et Paris) Histoire Urbaine $N^{\circ} 6$ E N S A De Paris-La Villette.

Delamare, Al. (1860). "Exploration Scientifique De L'algérie Pendant Les Années 1840-1845". Archéologie.

Despois, J., Raynal,R. (1967). Géographie De L'afrique Du Nord-Ouest, Paris, pp. 62-65.

Feraud, C. (2009). Histoire Des Villes De La Province De Constantine, Gigelly, Alger-Paris, 1870.

Granger, S. (1986). “Au Coeur Des Babors :Djidjelli” En Petite Kabylie, T.1, Paris.

Hannouf, F. (2009). Intervention Du Genie Militaire Français Sur Une Ville D'algerie Au Debut De La Colonisation:Djidjelli, 1839-1862. Mémoire De Master, Ensa De Paris-Belleville,

Koumas, A., Nafa, C. (2003). L'algérie Et Son Patrimoine, Dessins Français Du Xixe Siècle, Editions $\mathrm{Du}$ Patrimoine.

Lerma, J.L., Vidal, J., Portales, C. (2004). "Three-Dimensional City Model Visualisation For Real-Time Guided Museum Tours", Photogrammetric Record, Vol 19, № 106.

Malverti, X. (1994). "Les officiers du Génie et le dessin de villes en Algérie (1830-1870)", Revue du monde musulman et de la Méditerranée, $\mathrm{N}^{\circ} 73-74$. Figures de l'orientalisme en architecture. pp. 229244.

Oulebsir, N. (2004). Les Usages Du Patrimoine. Monuments, Musées Et Politique Coloniale En Algérie (1830-1930), Paris.

Retout, A. (1927). Histoire De Djidjelli, Alger.

Rozo-Ortega, K. (2009). Du Plan Au Volume. Mémoire De Master, E. N. S. A. De Nancy

Salama, P. (1974). « Igilgili, Vingt-Trois Siècles D'histoire », El Djezaïr, Revue De L'office National Du Tourisme Algérien, $\mathrm{N}^{\circ} 15$. 